\title{
Iterative Solution of Composite Problems with the Combined-Field Integral Equation
}

\author{
Özgür Ergül ${ }^{1}$ and Levent Gürel ${ }^{1,2}$ \\ ${ }^{1}$ Department of Electrical and Electronics Engineering \\ ${ }^{2}$ Computational Electromagnetics Research Center (BiLCEM) \\ Bilkent University, TR-06800, Bilkent, Ankara, Turkey \\ E-mail: ergul@ee.bilkent.edu.tr, lgurel@bilkent.edu.tr
}

\begin{abstract}
We consider the solution of electromagnetic problems related to microwave applications involving composite geometries with coexisting open and closed conductors. Combined-field integral equation is introduced on the closed parts of the geometry to improve the iterative solutions. It is demonstrated that the convergence rates are significantly increased compared to the conventional formulation with the electric-field integral equation.
\end{abstract}

Index Terms - Integral equations, iterative methods, multilevel fast multipole algorithm.

\section{INTRODUCTION}

Microwave applications often involve both thin and thick conductors in the same electromagnetic environment. An example is depicted in Fig. 1, where a full-wave dipole antenna is placed near a large rectangular platform. In the simulation environment, thin and thick conductors are modelled with open and closed surfaces, respectively. Then, the conventional solutions are restricted to employ the electric-field integral equation (EFIE) [1] due to the open parts of the geometry, since the magnetic-field integral equation (MFIE) [2], and therefore, the combined-field integral equation (CFIE) [3] are not applicable to open surfaces. Unfortunately, EFIE usually gives ill-conditioned matrix equations, especially when the geometry of the problem involves closed surfaces. With EFIE, as the problem size gets larger and iterative methods are employed, solution of the composite problems with coexisting open and closed surfaces becomes extremely difficult, even when the matrix-vector products are accelerated by the multilevel fast multipole algorithm (MLFMA) [4].

In this paper, we report an improved solution of the composite-type radiation problems by including the combinedfield integral equation in the formulation of the closed parts. Independent from the type of the iterative algorithm, the resulting hybrid formulation, i.e., the CFIE/EFIE formulation, consistently gives lower number of iterations compared to the conventional EFIE formulation. Convergence plots will be presented for the problem in Fig. 1, and the hybrid formulation can be easily extended to other composite-type problems involving various relative amounts of open and closed parts.

\section{CFIE Formulation of Composite Problems}

For conducting surfaces, EFIE and MFIE can be written in phasor notation with the $e^{-i w t}$ convention as

$$
\hat{\boldsymbol{t}} \cdot \int_{S} d \boldsymbol{r}^{\prime} \boldsymbol{J}\left(\boldsymbol{r}^{\prime}\right) \cdot\left(\overline{\mathbf{I}}-\frac{\nabla \nabla^{\prime}}{k^{2}}\right) g\left(\boldsymbol{r}, \boldsymbol{r}^{\prime}\right)=\frac{i}{k \eta} \hat{\boldsymbol{t}} \cdot \boldsymbol{E}^{i}(\boldsymbol{r})
$$
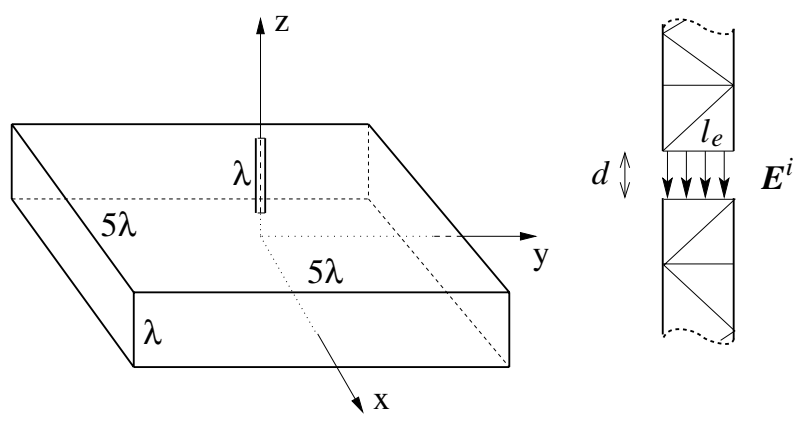

Fig. 1. (a) Radiation problem involving a dipole antenna (open surface) of length $\lambda$ located over a perfectly conducting rectangular box (closed surface) with the dimensions of $\lambda \times 5 \lambda \times 5 \lambda$. (b) Delta-gap source located at the center of the antenna.

and

$$
\boldsymbol{J}(\boldsymbol{r})-\hat{\boldsymbol{n}} \times \int_{S} d \boldsymbol{r}^{\prime} \boldsymbol{J}\left(\boldsymbol{r}^{\prime}\right) \times \nabla^{\prime} g\left(\boldsymbol{r}, \boldsymbol{r}^{\prime}\right)=\hat{\boldsymbol{n}} \times \boldsymbol{H}^{i}(\boldsymbol{r}),
$$

directly from the boundary conditions for the tangential electric and magnetic fields, respectively. In (1) and (2), scattered fields are expressed in terms of the induced (unknown) surface current $\boldsymbol{J}(\boldsymbol{r})$ with the aid of the free-space Green's function

$$
g\left(\boldsymbol{r}, \boldsymbol{r}^{\prime}\right)=\frac{e^{i k R}}{4 \pi R} \quad\left(R=\left|\boldsymbol{r}-\boldsymbol{r}^{\prime}\right|\right),
$$

where $r$ is an observation point on the surface, and $\hat{t}$ and $\hat{\boldsymbol{n}}$ are the tangential and normal vectors, respectively, at the observation point. On the right-hand sides of (1) and (2), $\boldsymbol{E}^{i}(\boldsymbol{r})$ and $\boldsymbol{H}^{i}(\boldsymbol{r})$ denote the incident electric and magnetic fields, respectively.

CFIE is obtained by the combination of EFIE and MFIE as

$$
\mathrm{CFIE}=\alpha \mathrm{EFIE}+(1-\alpha) \frac{i}{k} \mathrm{MFIE},
$$

where $k$ is the wavenumber associated with the operation frequency and $\alpha$ is a parameter between 0 and 1 . In a composite problem, we apply EFIE in (1) on the open parts, while CFIE with $\alpha=0.2$ is applied on the closed parts to improve the iterative solution. We note similar extended applications for CFIE in the literature, such as [5], where the authors aim to reduce the computation cost of the system formation, and [6], where the hybrid formulation is applied on thin and thick conductors that are completely closed.

\section{Results}

In Fig. 1(a), a full-wave dipole antenna is placed near a large rectangular conducting platform with dimensions $\lambda \times 5 \lambda \times 5 \lambda$. 
The dipole antenna is modelled by a strip of zero thickness with length $\lambda$ and width $\lambda / 10$. The composite structure is triangulated with a mesh size of $\lambda / 10$ corresponding to 16,124 triangles on the box and only 20 triangles on the strip. We employ the Rao-Wilton-Glisson [7] functions as the basis and testing functions defined on the triangular domains. The resulting linear system involves a dense matrix equation with 24,205 unknowns. The feed of the antenna is simulated by a delta-gap source located at the center of the strip as shown in Fig. 1(b).

As depicted in Fig. 2, the radiation problem in Fig. 1 is solved by various iterative methods [8] employing different formulations. Independent from the type of the iterative method, the hybrid CFIE/EFIE formulation obtained by employing CFIE on the closed box, has significantly improved convergence rates compared to the conventional EFIE formulation. For comparisons, the figures also include the hybrid MFIE/EFIE formulation obtained by employing MFIE on the closed parts, which gives slower convergence compared to the CFIE/EFIE formulation, but still better than the EFIE formulation.

\section{CONCLUSION}

Improved iterative solutions of composite-type problems involving both thin and thick conductors are reported. By employing CFIE on the thin conductors modelled by the closed surfaces, the convergence rates are significantly improved compared to the conventional EFIE formulation.

\section{ACKNOWLEDGEMENT}

This work was supported by the Turkish Academy of Sciences in the framework of the Young Scientist Award Program (LG/TUBA-GEBIP/2002-1-12), by the Scientific and Technical Research Council of Turkey (TUBITAK) under Research Grant 105E172, and by contracts from ASELSAN and SSM.

\section{REFERENCES}

11] A. W. Glisson and D. R. Wilton, "Simple and efficient numerica methods for problems of electromagnetic radiation and scattering from surfaces," IEEE Trans. Antennas Propagat., vol. AP-28, no. 5, pp. 593 603, Oct. 1980.

[2] A. J. Poggio and E. K. Miller, "Integral equation solutions of threedimensional scattering problems," in Computer Techniques for Electromagnetics, R. Mittra, Ed. Oxford: Permagon Press, 1973, Chap. 4.

[3] J. R. Mautz and R. F. Harrington, "H-field, E-field, and combined field solutions for conducting bodies of revolution," $A E \ddot{U}$, vol. 32, no. 4 , pp. 157-164, Apr. 1978

[4] W. C. Chew, J.-M. Jin, E. Michielssen, and J. Song, Fast and Efficient Algorithms in Computational Electromagnetics. Boston, MA: Artech House, 2001.

[5] A. Helaly and H. M. Fahmy, "Combined-field integral equation," Electronics Lett., vol. 29, no. 19, pp. 1678-1679, Sept. 1993.

[6] W. V. T. Rusch and R. J. Pogorzelski, "A mixed-field solution for scattering from composite bodies," IEEE Trans. Antennas Propagat., vol. 34, no. 7, pp. 955-958, July 1986

[7] S. M. Rao, D. R. Wilton, and A. W. Glisson, "Electromagnetic scattering by surfaces of arbitrary shape," IEEE Trans. Antennas Propagat., vol. AP-30, no. 3, pp. 409-418, May 1982.

[8] S. Balay, K. Buschelman, V. Eijkhout, W. D. Gropp, D. Kaushik, M. G. Knepley, L. C. McInnes, B. F. Smith, and H. Zhang, PETSC Users Manual, Argonne National Laboratory, 2004.
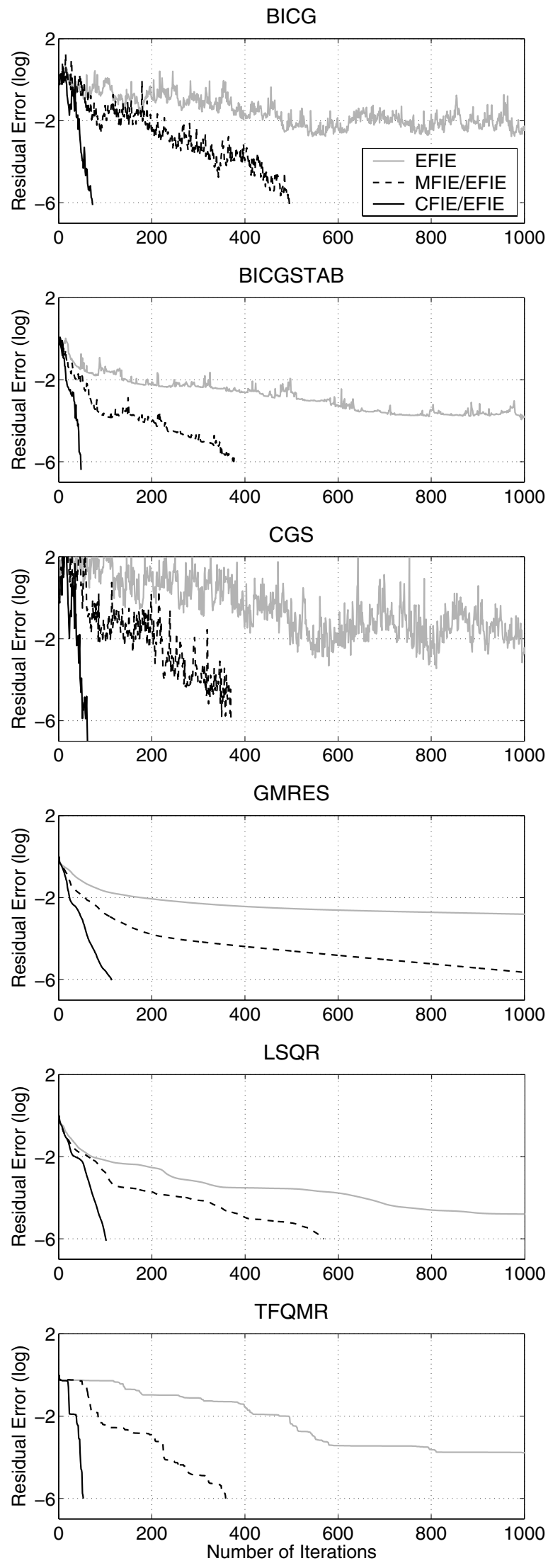

Fig. 2. Convergence characteristics of the problem in Fig. 1(a) for various iterative methods and different formulations, i.e., EFIE, MFIE/EFIE, and CFIE/EFIE. 